\title{
Die neue EU-Mobilität: Gewinn für alle oder soziale Erosion?
}

\author{
DIETRICH THRÄNHARDT
}

\section{Migration in der offenen EU: ein gigantisches Experiment}

Die Europäische Union (EU) hat sich im Lissabonner Vertrag 2009 zum Ziel gesetzt, der wettbewerbsfähigste wissensbasierte Raum der Welt zu werden. Zugleich will sie die soziale Kohäsion wahren und stärken. Die EU-Bürger haben das Recht auf freie Mobilität in einem Raum von 500 Mio. Menschen. Zugleich wird damit die optimale Allokation des Faktors Arbeit im größten Wirtschaftsraum der Welt möglich. Während weltweit nur Güter, Kapital und zunehmend auch Dienstleistungen frei transferierbar sind, ist einer der Grundpfeiler der EU auch die freie Mobilität der Arbeitskräfte und der Menschen insgesamt.

Mit den Erweiterungen um Polen, die Tschechische Republik, die Slowakei, Ungarn, Slowenien und die Baltischen Staaten (E8) 2004 und um Rumänien und Bulgarien (E2) 2007 kamen Länder in die EU, die weit niedrigere Pro-KopfEinkommen haben als die alten Mitgliedstaaten. Schweden verdienen durchschnittlich etwa acht Mal so viel wie Bulgaren. Während Deutschland die Übergangsfristen voll ausnutzte, öffneten einige EU-Staaten ihre Arbeitsmärkte sofort. Damit entstand eine experimentelle Situation, aus der wir Rückschlüsse zum Verhältnis von Arbeitsund Sozialordnung und Migration ziehen können.

In der ersten Runde 2004 öffneten Schweden, Großbritannien und Irland ihre Arbeitsmärkte. In Schweden stieg die Zuwanderung aus Polen daraufhin mäßig von 1.134 Personen 2003 bis auf 7.540 Personen 2007 und ging dann wieder leicht zurück. Großbritannien dagegen erlebte eine starke Einwanderungswelle, weit mehr als die Regierung erwartet hatte. Heute leben 520.000 Polen in Großbritannien, 400.000 von ihnen sind beschäftigt, ein außerordentlich hoher Anteil. ${ }^{1}$ In der zweiten Runde 2007 finden wir ähnliche Diskrepanzen. Spanien, Italien, Portugal und vier skandinavische Staaten öffneten ihre Arbeitsmärkte. Die Einwanderung aus Rumänien nach
Schweden war wiederum moderat. Sie nahm von 348 Personen 2006 bis auf 2.449 Personen 2008 $\mathrm{zu}$ und fiel dann wieder. Auf der anderen Seite finden wir heute je eine Million Rumänen in Italien und in Spanien - die größten Wanderungsbewegungen zwischen einzelnen Ländern innerhalb der EU überhaupt.

\section{Regulierung in Schweden, Deregulierung in Großbritannien}

Nach seinen positiven Erfahrungen mit einem offenen Modell der Einwanderung aus der EU öffnete Schweden seinen Arbeitsmarkt 2008 weltweit. Jeder Mensch, der einen Arbeitsplatz in Schweden findet oder angeboten erhält, kann nach Schweden kommen und dort arbeiten - immer vorausgesetzt, dass der tarifliche Lohn bezahlt wird. Diese weltweit einmalige Arbeitsmarktöffnung wird in der internationalen Diskussion zu wenig beachtet, wohl weil sie keine Dramatik auslöste. Die generelle Durchsetzung der Geltung des Tarifsystems, begleitet von allgemeiner Offenheit über die Verdienste in Schweden und einem handlungsfähigen Staat, gibt Unternehmen keine Anreize und Möglichkeiten, Migranten deshalb einzustellen, um sie zu geringeren Löhnen oder schlechteren Bedingungen arbeiten zu lassen. Die Einwanderer aus Polen verdienen im Durchschnitt etwa so viel wie ihre schwedischen Kollegen und werden weitgehend entsprechend ihren Qualifikationen beschäftigt.

Auf der anderen Seite führten die vielen Einwanderer aus den Beitrittstaaten nach Großbritannien und in die südlichen EU-Länder zu zusätzlichem Druck auf die niedrigen Einkommen und zu Dequalifikation, was in der Migrationsforschung als „brain waste“ bezeichnet wird. In Großbritannien arbeiten EU-Einwanderer „for low wages in low-skill jobs, even if they are highly educated, [...] across the country in diverse and dispersed locations". ${ }^{2}$ Die Wirtschaft ist dereguliert und die Spreizung der Einkommen nimmt stark zu. Einwanderung ist nicht der Hauptgrund dafür, aber sie ist diesem Prozess ausgeliefert und verstärkt ihn. Im politischen Diskurs in Großbritannien dient sie als Sündenbock für Probleme aller Art.

\section{Migration in die Schattenwirtschaft und in den „mediterranen Sozialstaat“}

In Italien und in Spanien sind deregulierte Situationen und die Schattenwirtschaft noch bestimmender. Migrantinnen arbeiten vor allem in der Pflege und im Haushalt, Migranten in der Bauindustrie. Auch in der Wirtschaftskrise seit 2007 setzte sich die Einwanderung vor allem in diese Sektoren fort, trotz steigender Arbeitslosigkeit insgesamt. In der Krise waren ungeschützte Migranten für viele Firmen besonders interessant, es kam zu einer „compression of labour costs, translated into an enduring demand for cheap, foreign labour". ${ }^{3}$ Gleichzeitig verschlechterte sich die ökonomische Situation in Herkunftsstaaten wie Rumänien. Deswegen wuchs das Bedürfnis nach Auswanderung und inzwischen aufgebaute Netzwerke erleichterten Kettenmigration. Drei Viertel der Arbeitskräfte in Haushalten sind inzwischen Ausländer, ganz überwiegend Frauen. Der Bereich häuslicher Dienstleistungen entwickelte sich zu einer Nische mit niedrigen

1 Wadensjö, E./Ruhs, M./Wójcicka, A. (2012): Labour migration. What's in it for us?, Stockholm.

2 Sumption, M./Somerville, W. (2010): The UK's new Europeans. Progress and challenges five years after accession,

http://www.equalityhumanrights.com/uploaded files/new_europeans.pdf.

3 Sciortino, G. (2013): Immigration in Italy: Subverting the logic of the welfare reform?, in: Brochmann, G./Jurado, E. (Hrsg.): Europe's immigration challenge. Reconciling work, welfare and mobility, New York, S. 77-94. 
Löhnen und problematischen Arbeitsbedingungen - bei wenig staatlicher Kontrolle. Deswegen werden diese Berufe von Einheimischen mehr und mehr gemieden.

In Italien ist ein Teil der Arbeitsverhältnisse stark reglementiert und geschützt, vor allem im Staatssektor und bei den großen Firmen, davon profitieren vor allem ältere Einheimische. Andere Sektoren bleiben unreguliert und sind von geringen Löhnen und unsicheren Arbeitsverhältnissen geprägt. Schätzungsweise $17 \%$ der Wirtschaftsleistungen werden im informellen Sektor erzeugt, also ohne Steuern und Sozialabgaben. Im Unterschied etwa zu Skandinavien stellt der Staat wenig öffentliche Dienstleistungen wie Kindergärten und Pflegeeinrichtungen zur Verfügung. Der „mediterrane Sozialstaat“ ist durch staatliche Geldleistungen geprägt: hauptsächlich Renten, überwiegend für ältere Menschen. Die Versorgung von Kindern, Alten und Pflegebedürftigen obliegt den Familien, mit der Folge niedriger Entlohnungen für Haushaltshilfen und häusliche Angestellte.

Während in Italien also die Einwanderung trotz einer stagnierenden Wirtschaftssituation in vermindertem Ausmaß weitergeht, ist Großbritannien von Einwanderung in einer wachsenden Wirtschaft geprägt. Trotz eines seit fünf Jahren wiederholten Versprechens der Regierung, die Netto-Einwanderung auf unter 100.000 Menschen zu senken, ist sie im Jahr 2014 auf die Rekordzahl von 318.000 Menschen angestiegen. Gleichzeitig sind die Löhne seit 2008 gesunken, bei den jüngeren Beschäftigten um 11 \%.

\section{Deutschland: Deregulierte Nischen in der sozialen Marktwirtschaft?}

In Deutschland sind zentrale Industriebereiche nach wie vor reguliert und die Gewerkschaften konnten in den letzten Jahren wieder Lohnsteigerungen durchsetzen. In vielen Service-Bereichen sind die gewerkschaftlichen Organisationsraten dagegen niedrig. Die Mehrzahl der EU-Zuwanderer hat Zugang zu regulären Jobs mit sozialer Absicherung. In der wachsenden Wirtschaft seit 2010 fanden Zuwanderer leicht Anstellungen und ohne sie wäre das Wachstum an Grenzen gestoßen. Ihr Verdienst und ihr Status in den Betrieben bleiben allerdings hinter denen ihren deutschen Kollegen zurück. ${ }^{4}$ Da das einheimische Arbeitskraftpotenzial zu schrumpfen beginnt, werden erste Erfahrungen mit einer neuen Konstellation gemacht: Zuwanderung als notwendige Bedingung zur Aufrechterhaltung der Leistungsfähigkeit der Wirtschaft und ihrer
Weiterentwicklung. Diese Situation trifft zurzeit nur für Deutschland zu, wird in Zukunft aber auch in anderen EU-Staaten und in der EU insgesamt gelten.

In einigen Branchen und Sektoren haben sich allerdings zugleich Konstellationen entwickelt, in denen Zuwanderer aufgrund ihrer mangelnden Kenntnis ihrer Rechte und ihrer sozialen Absicherung ausgebeutet werden. Die Fleisch- und Wurstindustrie, das Gastgewerbe und der Pflegebereich sind Beispiele, die mit Recht skandalisiert worden sind. Mehr Kontrollen und die Einführung des Mindestlohns können zu Behebung der Missstände beitragen. Problematisch bleiben gleichwohl die Auswirkungen der vielfältigen Möglichkeiten, Teilproduktionen auszugliedern („outsourcing“) und die Verantwortlichkeiten für die Zahlung von Löhnen und die Arbeitsbedingungen zu verlagern und zu verwischen. Deutschland bleibt zwar ganz überwiegend ein Land mit sozialen Standards und regulierten Arbeitsbedingungen, aber in der Verbindung von Deregulierung und Transnationalität konnten sich Ausbeutungsstrukturen entwickeln, die schwer wieder in den Griff zu bekommen sind. Die Klage der belgischen Regierung gegen die unfairen und unzumutbaren Bedingungen in der deutschen Fleischindustrie zeigen, wie dynamisch diese Prozesse die Arbeitsmärkte beeinflussen können, in Richtung eines „race to the bottom“ für Löhne und Arbeitsbedingungen. Auch in einem relativ wohlgeordneten Land wie Deutschland können schnell Nischen entstehen, in denen extreme Ausbeutungsverhältnisse mit großen Gewinnmargen verbunden werden.

\section{Regulierung reduziert Migrations- probleme, Deregulierung steigert sie}

Wenn wir die geschilderten Fälle vergleichen, erkennen wir, dass Mobilität im offen transnationalen Raum der EU nicht einfach von der Öffnung oder Schließung der Grenzen abhängt, wie es die öffentliche Diskussion oder einfache „PushPull-Modelle" nahelegen. Die Unterschiede in den Pro-Kopf-Einkommen und Lebensstandards sind zwar ein wesentliches Motiv für Wanderungen zwischen Ländern und Regionen, können aber das unterschiedliche Ausmaß in den unterschiedlichen Ländern auch nicht erklären. Es geht auch nicht, wie zur Zeit von der britischen Regierung propagiert, um die Höhe von Sozialleistungen, denn sonst müssten sehr viele Migranten gerade nach Schweden und in andere skandinavische Länder strömen. Es ist in diesem $\mathrm{Zu}$ sammenhang paradox, dass Sozialmissbrauch von Migranten gerade in Ländern diskutiert wird, in denen die Sozialleistungen gering sind - ein Hinweis darauf, dass etablierte Diskurse über "undeserving poor" an neuen Beispielen weitergeführt werden. Vielmehr werden Ausmaß und Qualität von Migrationen stark von den Arbeitsregimen der verschiedenen Länder bestimmt. ${ }^{5}$ Innere Kontrollen, insbesondere die Einhaltung von Standards der Beschäftigung und der Bezahlung sind von ausschlaggebender Bedeutung und sie sind weit effektiver als Grenzkontrollen. Wenn Standards allgemein akzeptiert sind und am Arbeitsplatz kontrolliert werden, durch Kolleginnen und Kollegen, Gewerkschaften, Betriebsräte oder auch konkurrierende Firmen, so ist dies weit wirksamer als die Kontrolle nur durch bürokratische Instanzen wie etwa die Zollbehörden. In Schweden kommt die öffentliche Transparenz in Bezug auf Informationen über Gehälter, Einkommen und Steuern hinzu. Die Löhne von Migranten werden in dieses System integriert, mit dem Ergebnis, dass in Schweden die Einkommen von EU-Einwanderern denen von Einheimischen gleichgestellt sind.

Auch in der Schweiz werden die Migranten in das Einkommensgefüge integriert, wenn auch in anderer Weise. Aus Anlass der Öffnung zur Freizügigkeit mit der EU hat die Schweiz „flankierende Maßnahmen“ ergriffen, darunter ein Entsendegesetz, das Lohndumping verunmöglicht, und die Allgemeinverbindlicherklärung von Gesamtarbeitsverträgen erleichtert bzw. Mindestlöhne vorsieht. Zur Überwachung wurden „Tripartite Kommissionen“ aus Arbeitgebern, Arbeitnehmern und Behördenvertretern geschaffen. Auf diese Weise wurde erreicht, dass das hohe Lohnniveau durch die Migration nicht ausgehebelt wurde, auch im Interesse der schweizerischen Betriebe. Zuwanderer aus der EU erreichen etwa das gleiche Einkommensniveau wie die Einhei-

4 Burkert, C. (2015): Europa auf Wanderschaft: Gelingende Arbeitsmarktintegration von europäischen Zuwanderergruppen am deutschen Arbeitsmarkt?, in: Pfeffer-Hoffmann, C. (Hrsg.): Profile der Einwanderung. Differenzierungen in einer emergenten Realität der Flüchtlings- und Arbeitsmigration (im Erscheinen).

5 In der Diskussion wird oft auf die sprachliche Nähe des Rumänischen zum Italienischen und Spanischen verwiesen. Für die Migrantinnen und Migranten ist dies sicherlich eine Erleichterung. Die problemlose Einwanderung auch aus der Ukraine und vieler anderer Gruppen nach Italien und Spanien zeigt aber, dass dies nur ein Nebenaspekt ist. 
mischen. Die Schweiz steigert ihre Wettbewerbsfähigkeit durch diese Art der Einwanderungspolitik erheblich, sie zieht qualifizierte Spezialisten an und setzt sie entsprechend ihrer Qualifikationen ein - ganz im Gegensatz zu früheren Perioden, in denen Einwanderer für unqualifizierte Tätigkeiten angeworben wurden.

Neben den Standards bei Löhnen und Gehältern spielt die Konstruktion des Sozialstaats eine große Rolle bei der Gestaltung der innereuropäischen Mobilität. Wenn Kinderbetreuung, Erziehung, Altenpflege und die Fürsorge für Behinderte befriedigend durch staatliche Leistungen oder verpflichtende Versicherungssysteme geregelt sind, entstehen anständige Berufsmöglichkeiten. Insgesamt ist dies ein sehr großer und vielfältiger Dienstleistungsbereich, der in Skandinavien am weitgehendsten ausgebaut ist. Ist er von den Arbeitsbedingungen her attraktiv ausgestattet, so wird er durchaus von Einheimischen angenommen und Migrantinnen finden Arbeitsmöglichkeiten und eine befriedigende Arbeitssituation. Sind diese Bereiche dagegen nicht sozialstaatlich durchfinanziert, lückenhaft ausgebaut oder qualitativ inakzeptabel, sowohl was die Versorgung wie was die Berufssituation der Beschäf- tigten angeht, so entsteht ein Bedarf nach kostengünstigen Kräften, die überwiegend von Familien angestellt werden, und zwar zu sehr unterschiedlichen Bedingungen, vom Familienanschluss bis zur völligen Rechtlosigkeit. Dieser Zustand ist typisch für Italien und andere Mittelmeerländer. In Deutschland ist er verbreitet bei der Pflege von älteren Menschen. Hier hat sich eine deutsch-polnische Vermittlungsbranche etabliert, die mit dem Angebot wirbt, dass polnische Pflegekräfte sieben Tage lang 24 Stunden zur Verfügung stehen.

Die EU etabliert einen offenen Arbeitsmarkt und sie sichert die freie Mobilität. Aber die Qualität der Beschäftigungsverhältnisse und der beruflichen und sozialen Integration und die daraus folgende politische Wahrnehmung und Artikulation werden nach wie vor national und regional geformt. Entscheidend sind die Mechanismen der Regulierung des Arbeitsmarktes und der sozialen Versorgung. Die Quantität und Qualität der Einwanderung kann wiederum dazu beitragen, die entsprechenden Strukturen weiter zu verstärken oder zu verformen, indem sie entweder zu mehr Offenheit und einem erweiterten Verständnis von Solidarität beitragen oder Un- terschiede noch deutlicher und sichtbarer machen, indem soziale Diskrepanzen kulturalisiert und bestimmten Einwanderungsgruppen oder Ethnien zugeschrieben werden. Insofern ist Einwanderung eine Herausforderung und eine Chance - für die sozialen Verhältnisse, das Ausmaß der Gleichheit oder Ungleichheit ebenso wie für die Effizienz und Wettbewerbsfähigkeit der Einwanderungsländer und der EU insgesamt. Mobilität kann zu mehr Lebensqualität und Wettbewerbsfähigkeit beitragen, wenn die Migranten entsprechend ihren Fähigkeiten arbeiten können und gleichberechtigt in die Wirtschaft und Gesellschaft integriert werden.

\section{AUTOR}

DIETRICH THRÄNHARDT, Professor em. für Politikwissenschaft, insbesondere Vergleichende Migrationsforschung an der Westfälischen WilhelmsUniversität Münster.

thranha@uni-muenster.de 